\title{
Structure of thallium and lead calculated from Shaw local pseudo- potential and molecular dynamics
}

\author{
B. Grosdidier ${ }^{1}$,D. Es Sbihi ${ }^{1}$, A. Ben Abdellah ${ }^{1,2}$, S. Hellal $^{3}$ and J. G. Gasser ${ }^{1}$
}

\author{
${ }^{1}$ Laboratoire de Physique des Milieux Denses, Institut de Chimie, Physique et Matériaux, Paul Verlaine - Metz. 1, Bd D. F. \\ Arago 57078 Metz Cedex 3, France ( E-mail grosdidi@univ-metz.fr ) \\ ${ }^{2}$ Groupe de Recherche de Physique-Mécanique, Université Abdelmalek Essaadi, Faculté des Sciences et Techniques de \\ Tanger B.P. 416 Tanger, Maroc \\ ${ }^{3}$ Laboratoire de Physique et Chimie Quantique - Hasnaoua, Université Mouloud Mammeri de Tizi-Ouzou, 15000, Algeria
}

\begin{abstract}
Recently, we (Es Sbihi Phil. Mag 2010) have successfully calculated, by molecular dynamics, the static structure factor of liquid bismuth at different temperatures. Our results were in very good agreement with the Waseda experimental data. Our assumption was to consider the true density of states which presents a gap as measured by Indlekofer (J. Non-Cryst. Solids 1989) and calculated by Hafner-Jank (Phys. Rev. B 1990) for liquid bismuth. The number of electrons at the Fermi energy has been calculated with three conduction electrons for bismuth (number of $\mathrm{p}$ electrons). With this assumption, the structures were determined with an effective ion-ion potential constructed from the Shaw local Optimised Model Potential (OMP) and the Ichimaru-Utsumi dielectric function. In the present paper, we generalize our assumptions to liquid thallium and lead which also present such a gap. Their calculated structures are also very close to the experimental ones. This confirms that the number of conduction electrons on the Fermi sphere is consistent with the number of $p$ electrons as has been even shown for our electronic transport properties of liquid lead (A. Ben Abdellah, Phys. Rev. B 2003).
\end{abstract}

\section{Introduction}

The bismuth-lead alloy serves as cooling fluid in nuclear reactors [1]. The knowledge of the physical properties of heavy metals is crucial for this technology [2]. In this paper we extend our new approach already used for bismuth [3] to others heavy metals as thallium and lead. This approach used in our previous work [3], was to consider the Density Of States (DOS) which presents a gap as measured by Indlekofer [4] and calculated by Hafner and Jank [5] for liquid bismuth. The number of electrons at the Fermi energy has been calculated with three conduction electrons for bismuth (number of $\mathrm{p}$ electrons). With this assumption, the structures have been determined with an effective ion-ion potential constructed from the Shaw [6] local Optimised Model Potential (OMP) and the Ichimaru-Utsumi [7] dielectric function. In the present paper, we apply our assumptions to liquid thallium and lead which also present such a gap as well as on the experiment [4] or on Hafner-Jank [8,9] calculated DOS. The experimental and calculated DOS are displayed in Figure 1. We show that their calculated ionic structures match very well the experimental ones. Furthermore, our assumption confirms that the number of conduction electrons on the Fermi sphere is consistent with the number of $p$ electrons as has been even shown for our electronic transport properties of liquid thallium and lead [10]. The ionic structure calculation was performed by molecular dynamics (MD) on a 4000 particles NVT system. Our calculated pair correlation functions and structure factors are compared to the experimental ones (Waseda [11]). The paper is written as it follows. In the second theoretical part we present briefly the calculation of the ion-ion effective potential obtained from OMP pseudopotential and the numerical code which calculates the structure from these effective potentials. The results are presented and discussed in the third part. We show the very good agreement between the experimental structure factors and the calculated ones for liquid thallium and lead with OMP and respectively one and two conduction electrons. We also discuss the coherence with the electronic transport properties such as the resistivity. Finally, we conclude. (Unless explicitly stated otherwise, atomics units are used throughout: $\hbar=e=m=1$ ). 


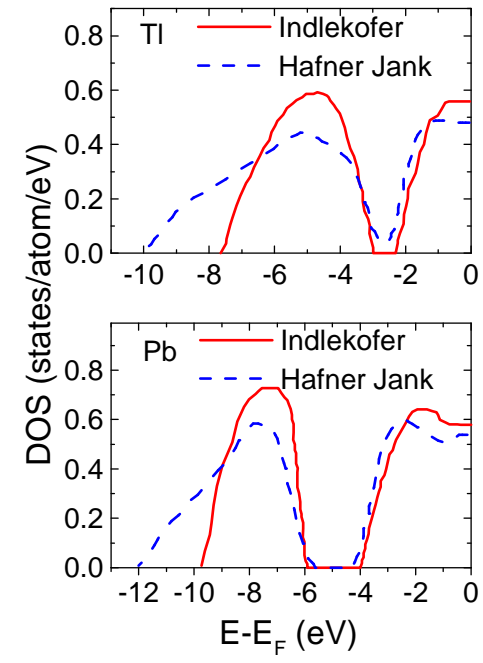

Fig. 1: Indlekofer [4] experimental (full line) and Hafner Jank $[8,9]$ calculated (dashed line) DOS of thallium and lead.

\section{Theoretical tools}

\subsection{Effective potential}

The pseudo-potential theory is synthesised in the reference [12]. The expression of the ion-ion effective potential $V(r)$ calculated from the pseudo-potential is given by:

$$
V(r)=\frac{Z^{2}}{r}\left(1-\frac{2}{\pi} \int_{0}^{\infty} F^{N}(q) \frac{\sin (q r)}{q} d q\right) .
$$

Here the number $Z$ is the chemical valence. The quantity $r$ is the inter-ionic distance. The normalized energy wave number characteristic $F^{N}(q)$ in reciprocal space, is defined from the Fourier transform of the local electronion model potential $W_{0}(q)$ :

$$
F^{N}(q)=\left(\frac{\Omega_{0} q^{2}}{4 \pi Z}\right)^{2} w_{0}(q)^{2} \frac{1}{1-G(q)}\left(1-\frac{1}{\varepsilon(q)}\right),
$$

where $\Omega_{Q}$ is the mean atomic volume. The quantity $\varepsilon(q)$ is the dielectric function, and the function $G(q)$ is the local field correction. This correction becomes more important when the electronic density increases. In this work, we choose the Ichimaru-Utsumi [7] (IU) expression that we used also for liquid bismuth. The expression of the local Shaw [6] Optimized Model Potential (OMP) is in the real space:

$$
w_{0}(r)=\left\{\begin{array}{cc}
A & r<R_{C} \\
-\frac{Z}{r} & r<R_{C}
\end{array},\right.
$$

where core radius $R_{C}$ is given by the optimization condition $\left(A=-Z / R_{C}\right)$. The Fourier transform $w_{0}(q)$ of $w_{0}(r)$ is expressed as:

$$
w_{0}(q)=-\frac{4 \pi Z}{\Omega_{0} q^{2}} \frac{\sin \left(q R_{C}\right)}{q R_{C}}=w_{0}^{O M P}(q)
$$

The previous equations clearly exhibit that the chemical valence $Z$ plays an essential role in the shape of the effective potential.

\subsection{Molecular dynamics}

The liquid metals structure is calculated by a simulation code. This latest uses the standard Verlet [13] algorithm and processes in NVT ensemble. The number of involved particles in the cubic simulation box is equal to 4000 . The side length is equal to $35 \AA$. The time step $\delta t$ is equal to $7 \cdot 10^{-15} s$ and the number of iterations after thermalization is equal to 30000 . The pair correlation functions $g(r)$ are calculated for each configuration and are meant. The reliable static structure factor $S(q)$ is obtained from the pair correlation function $g(r)$ by Fourier transform.

\section{Results and discussion}

For both heavy metals the density is calculated using Lucas [14] compilation of density as a function of temperature under the form: $d=a-b\left(T-T_{M}\right)$, where $T_{M}$ is the melting temperature of the metal. These parameters are given in Table 1.

Table 1. Density parameters [14] of the two heavy metals used for our calculations.

\begin{tabular}{|c|c|c|}
\hline Element & Thallium & Lead \\
\hline$T_{M}\left({ }^{\circ} \mathrm{C}\right)$ & 304 & 327.4 \\
\hline $\begin{array}{c}\text { Density value } a \\
\left(\mathrm{~g} \cdot \mathrm{cm}^{-3}\right)\end{array}$ & 11.29 & 10.665 \\
\hline $\begin{array}{c}\text { Density value } b \\
\left(\mathrm{~g} \cdot \mathrm{cm}^{-3} \cdot \mathrm{K}^{-1}\right)\end{array}$ & 14.80 & 12.64 \\
\hline$\rho_{0}\left(\mathrm{at} / \AA^{3}\right)=\Omega_{0}^{-1}$ & 0.03322 & 0.03095 \\
\hline
\end{tabular}

The effective potentials for liquid thallium and lead are calculated, from the OMP local pseudo-potential, with respectively valence values equal to 1 and 2 . All values needed for effective potentials calculations are given in the three first lines of Table 2.

Table 2. OMP potential parameters and coordination numbers of the two heavy metals.

\begin{tabular}{|c|c|c|}
\hline Element & Thallium & Lead \\
\hline Valence $Z$ & 1 & 2 \\
\hline Core radius $R_{C}(\AA)$ & 1.57 & 1.28 \\
\hline Temperature $\left({ }^{\circ} \mathrm{C}\right)$ & 315 & 340 \\
\hline $\begin{array}{c}\text { Our calculated } \\
\text { coordination } \\
\text { number }\end{array}$ & 12.40 & 11.29 \\
\hline $\begin{array}{c}\text { Our calculated } \\
\text { distance }(\AA)\end{array}$ & 4.44 & 4.40 \\
\hline $\begin{array}{c}\text { Waseda }[11] \\
\text { coordination } \\
\text { number }\end{array}$ & 11.6 & 10.9 \\
\hline
\end{tabular}

The calculated effective potentials are shown in Figure 2. 


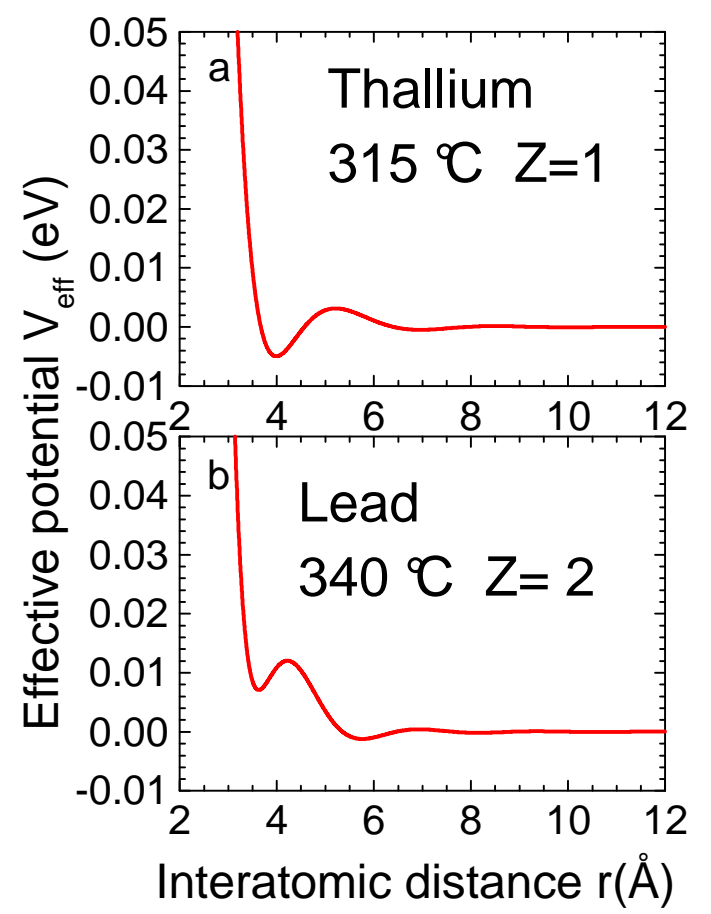

Fig. 2: Ion-ion effective potential for: (a) Thallium: $Z=1$, $T=315^{\circ} \mathrm{C}$ and (b): Lead: $Z=2, T=340{ }^{\circ} \mathrm{C}$.

These effective potentials are taken to calculate the pair correlation functions which are displayed in Figure 3 together with the Waseda's experimental ones.

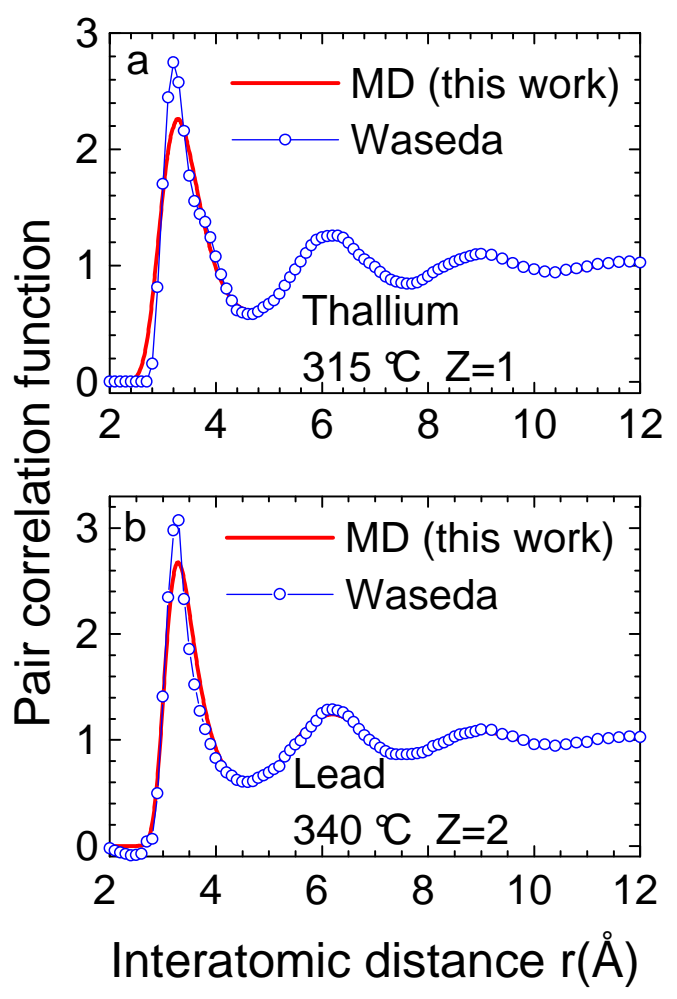

Fig. 3: Experimental [11] (circle) and computed (full line) pair correlation functions (a): for Thallium: $Z=1, T=315^{\circ} \mathrm{C}$ and (b): Lead: $Z=2, T=340^{\circ} \mathrm{C}$.
The structure factor are obtained by Fourier transform our calculated pair correlation functions and compared to Waseda's experimental ones (Figure 4).

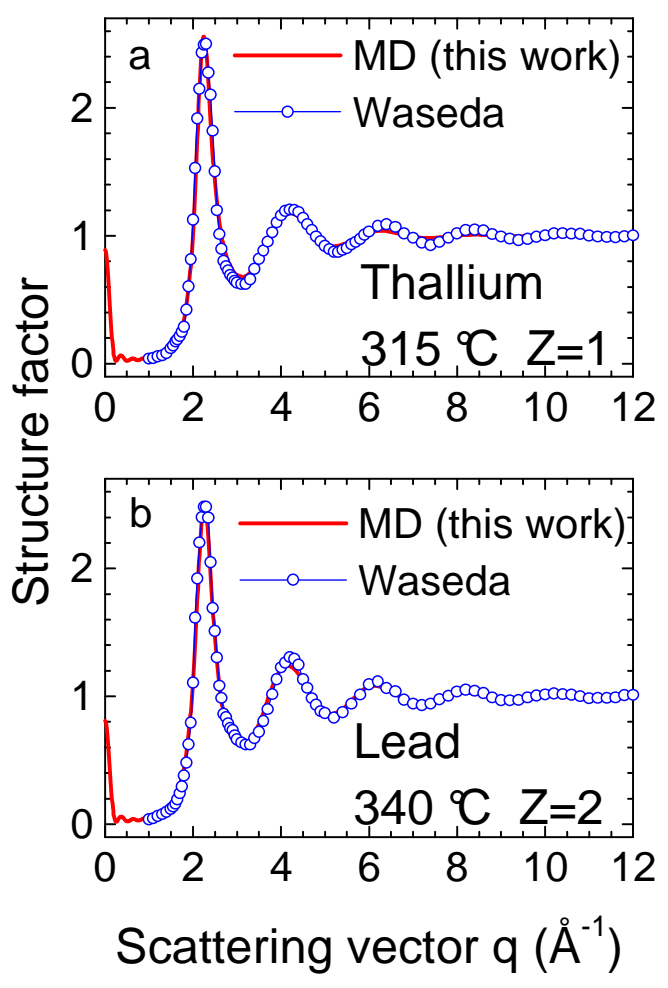

Fig. 4: Waseda [11] experimental structure factor (circle) and calculated static structure factors (full fine) for (a): Thallium: $Z=1, T=315^{\circ} \mathrm{C}$ and (b): Lead: $Z=2, T=340^{\circ} \mathrm{C}$.

For thallium, the effective potential presents a well (figure 2.a) which is revelatory of a high coordination number (11.6[11]) relatively to lead. The agreement between the calculated and experimental pair correlation functions is very good except on the height of the main peak. The agreement is better on the structure factors (figure 4.a) mainly on the first and second peaks. Our calculated coordination number (12.4) overestimated weakly ( $9 \%)$ the experimental one (11.6).

For lead the effective potential displays a pre-well (figure 2.b) which reveals a coordination number (11.29 [11]) smaller than to that of thallium. In figure 3.b, there is only a difference on the height of the first peak, but our results are a little better than those obtained by Witkowska et. al. [15] with an embedded atom method (EAM), especially on the positions of other extremes. Our calculated coordination number (11.29) is very close to the experimental one (10.9).

Our results bring improvements in regard to those obtained by Mendoub et. al. [16]. These authors determined the structure factor of liquid thallium with three conduction electrons and lead with four conduction electrons with the Fiolhais [17] pseudo-potential and molecular dynamics (MD) simulation. Their results, regarding the peaks positions, are quite satisfactory but discrepancies appear on their amplitudes. Our calculated values are in pretty good agreement both for the pair correlation functions and for the structure factors. 
The choice of the number of $\mathrm{p}$ electrons as the valence $Z$ considerably improves structure factor calculations. Kalhloukh et al. [18] showed that this choice provides better results for resistivity calculations of liquid bismuth. In an earlier paper we even proved that a valence 2 is a better choice for explaining the resistivity of liquid lead [10]. We check our choice by calculating the electrical resistivity for the liquid thallium. The determination of electrical resistivity is performed from the extended Ziman formalism $[19,20]$ which is non perturbative. For the two heavy metals we construct the muffin tin potentials necessary to the electrons scattering. The muffin tin potentials building following the method of Mukhopadhyay [21] is explained in reference [10]. We give our values of the resistivities for liquid thallium and liquid lead with the two sets of valences $(3,4)$ and $(1,2)$. All calculations are performed in the frame of the Esposito approach [22]. Our results are presented in Table 3 together with experimental values $[10,23]$ (third line of Table 3 .

Table 3. Liquid thallium and lead experimental (third column) and calculated electrical resistivity for $\mathrm{s}+\mathrm{p}$ conduction electrons (fourth line) and for $p$ conduction electrons (fifth line).

\begin{tabular}{|c|c|c|}
\hline Element & Thallium & Lead \\
\hline Temperature $\left({ }^{\circ} \mathrm{C}\right)$ & 315 & 750 \\
\hline $\begin{array}{c}\text { Experimental } \\
\text { resistivity } \rho(\mu \Omega \cdot \mathrm{cm})\end{array}$ & 73 [23] & $113.4[10]$ \\
\hline $\begin{array}{c}s+p \text { valence resistivity } \\
\rho(\mu \Omega \cdot \mathrm{cm})\end{array}$ & $\begin{array}{l}Z=3 \\
39.19\end{array}$ & $\begin{array}{c}Z=4 \\
39.37[10]\end{array}$ \\
\hline $\begin{array}{c}p \text { valence resistivity } \\
\rho(\mu \Omega \cdot \mathrm{cm})\end{array}$ & $\begin{array}{l}Z=1 \\
94.11\end{array}$ & $\begin{array}{c}Z=2 \\
68.8[10]\end{array}$ \\
\hline
\end{tabular}

It appears clearly that, the values calculated with only $p$ conduction electrons are always closer to the experimental resistivities. All these results confirm the extension our assumptions about the choice of the valences.

\section{Conclusion}

This work, which follows those regarding liquid bismuth, proves that calculations of properties of heavy liquid metals with the Shaw local pseudo-potentials and with the number of $\mathrm{p}$ electrons as valence give good results. These latest are better than those obtained earlier by with other local pseudo-potentials $[8,9,16]$ and with the usual values of the valences. This approach using the local OMP may serve for the study of other properties such as the dynamical structure factor and atomic transport properties (diffusion, viscosity...). These assumptions may also be used to calculate the structure of liquid bismuth-lead liquid alloy which had an increasing interest as coolant and spallation source in the conversion of radioactive element in accelerator-driven systems (ADS) or for nuclear reactor cooling.

\section{References}

1. B. F. Gromov, Yu. S. Belomitcev, E. I. Yefimov, M. P. Leonchuk, P. N. Martinov, Yu. I. Orlov, D. V. Pankratov, Yu. G. Pashkin, G. I. Toshinsky, V. V. Chekunov, B. A. Shmatko, V. S. Stepanov, Nucl. Eng. Des. 173, 207 (1997)

2. F. Sar, S. Mhiaoui and J. G. Gasser, J. Non-Cryst. Solids 353, 3622 (2007)

3. D. Es Sbihi, B. Grosdidier, A. Ben Abdellah and J. G. Gasser, Phil. Mag., 90, 1511 (2010)

4. G. Indlekofer, A. Pflugi, P. Oelhafen; J. NonCryst. Solids 117/118, 351 (1989)

5. J. Hafner and W. Jank, Phys. Rev. B 45, 2739 (1992)

6. R. W. Shaw, Phys. Rev. B 5, 4742 (1972)

7. S. Ichimaru and K. Utsumi, Phys. Rev. B 24, 7385 (1981)

8. J. Hafner and W. Jank, Phys. Rev. B 42, 11530 (1990)

9. W. Jank and J. Hafner, Phys. Rev. B 41, 1497 (1990)

10. A. Ben Abdellah, J. G. Gasser, A. Makradi, B. Grosdidier and J. Hugel, Phys. Rev., B 68184204 (2003)

11. Y. Waseda, "The Structure of Non-Crystalline Materials”, (McGraw-Hill, New York), (1980)

12. G. B. Bachelet, in: Strategies for Computer Chemistry (C. Tosi, Ed.), Kluwer Academic Publishers, Dordrecht (1989)

13. L. Verlet, Phys. Rev. 159, 98 (1967); Phys. Rev. 165, 201 (1968).

14. L. D. Lucas, "Techniques de l'Ingénieur, Traité des Matériaux Métalliques », Formulaire M65, France

15. A. Witkowska, J. Rybicki, S. De Panfilis, A. Di Cicco, J. Non-Cryst. Solids 352, 4351 (2006)

16. E.B. El Mendoub, R. Albaki, I. Charpentier, J.-L Bretonnet, J.-F. Wax et N. Jakse,J. Non-Cryst. Solids 3533475 (2007)

17. C. Fiolhais, J. P. Perdew, S. Q. Armster, J. M. Mac Laren and M. Brajczewska, Phys. Rev. B51 14001 (1995)

18. K. Khalouk, C. Chaib and J. G. Gasser, Phil. Mag., 89, 249 (2009)

19. J.M. Ziman; Phil. Mag. 6, 1013 (1961)

20. O. Dreirach, R. Evans, H. Güntherodt and H.-U. Kunzi, J. Phys. F2, 709 (1972)

21. G. Mukhopadhyay, A. Jain and V. K. Ratti; Sol. St. Com. 13, 1623 (1973)

22. E. Esposito, H. Ehrenreich and S. D. Gelatt, Phys. Rev. B 18, 3913 (1978)

23. M. Shomoji, "An introduction to the Physics and Chemistry of metals in the Liquid State", (Academic Press), (1977) 